\title{
Comparison of KRAS and PIK3CA gene status between primary tumors and paired metastases in colorectal cancer
}

This article was published in the following Dove Press journal:

OncoTargets and Therapy

20 April 2016

Number of times this article has been viewed

\author{
Qiong $\mathrm{He}^{1}$ \\ Qi $X u^{\prime}$ \\ Wei Wu' \\ Lei Chen' \\ Weijing Sun ${ }^{2}$ \\ Jieer Ying'
}

'Department of Chemotherapy, Zhejiang Cancer Hospital, Hangzhou, People's Republic of China;

${ }^{2}$ Department of Gastrointestinal Medical Oncology, School of Medicine, University of Pittsburgh, Pittsburgh, PA, USA
Correspondence: Jieer Ying Department of Chemotherapy, Zhejiang Cancer Hospital, 38 Guangji Road, Hangzhou, 310022 , People's Republic of China Tel $+8657 \mid 13858195803$ Fax +8657188122012

Email jieerying@aliyun.com

Weijing Sun

Department of Gastrointestinal Medical Oncology, School of Medicine, University of Pittsburgh, 1218 Scaife Hall, 3550 Terrace Street, Pittsburgh, PA I526I, USA

Email sunw@upmc.edu
Purpose: In metastatic or recurrent colorectal cancer (MRCRC), the concordance of Kirsten rat sarcoma viral oncogene homolog $(K R A S)$ and phosphatidylinositol-4,5-bisphosphate 3-kinase catalytic subunit alpha ( $P I K 3 C A)$ mutation status between the primary tumors and metastases is still controversial. The purpose of this study was to evaluate the association between KRAS and $P I K 3 C A$ mutational status and various clinicopathologic features, and compare their genotype in primary tumors with that of the paired metastatic tumors.

Method: We compared the mutation status of KRAS and PIK3CA between the primary tumors and the paired metastases of 59 MRCRC patients with available tissues (resection or biopsy). The presence of $K R A S$ and PIK3CA mutations were determined by direct sequencing analysis.

Results: Seventeen patients $(28.8 \%)$ had the KRAS mutation and 46 patients $(80.0 \%)$ had the $P I K 3 C A$ mutation when considering both the primary and metastatic sites. KRAS mutation was observed in ten primary tumors and eleven related metastases ( $16.9 \%$ vs $18.6 \%)$, while PIK3CA mutation was found in 26 primary tumors and 32 related metastases ( $44.1 \%$ vs $54.2 \%)$. KRAS status was concordant between primary and metastatic sites in 45 patients $(76.3 \%$, kappa $=0.157)$, while the concordance of $P I K 3 C A$ status was only found in 25 patients $(42.4 \%$, kappa $=-0.141)$. The PIK3CA status discordance rate was significantly higher in 40 patients undergoing metachronous resection of primary tumor or metastasis, compared with that in 19 patients with synchronous resection of primary tumor or metastasis (67.5\% [27/40] vs 36.8\% [7/19]; $P=0.026$ ).

Conclusion: Our results demonstrate that low concordance of $K R A S$ and high discordance of PIK3CA mutational status exist between the primary tumors and paired metastasis, and these findings remind us to have second thoughts about the need to evaluate metastatic tumors separately rather than only based on the primary tumor data when targeted therapy is considered.

Keywords: $K R A S, P I K 3 C A$, colorectal cancer, primary tumor, metastatic site

\section{Introduction}

Colorectal cancer (CRC) is the third most common cancer and the second leading cause of cancer-related deaths worldwide. Despite improvements made in therapy refinement, $25 \%$ of patients are diagnosed at IV stage of the disease, and approximately $50 \%$ of patients develop into metastatic or recurrent colorectal cancer (MRCRC) even though they received curative resection of primary CRC during the first 5 years from diagnosis. ${ }^{1}$ For MRCRC patients, systematic therapy containing chemotherapy, radiotherapy, and targeted therapy was considered to be the major treatment. As for approximately $60 \%-70 \%$ Kirsten rat sarcoma viral oncogene homolog $(K R A S)$ wild-type MRCRC, anti-epidermal growth factor receptor (anti-EGFR) inhibitors, such as cetuximab and 
panitumumab might enhance antitumor effects combined with chemotherapy according to recent guidelines. ${ }^{2}$ However, the response rate is not high, nearly $50 \% K R A S$ wild-type MRCRC patients cannot benefit from these combined therapies with anti-EGFR inhibitors. ${ }^{3-5}$ Emerging data have proposed phosphatidylinositol-4,5-bisphosphate 3-kinase catalytic subunit alpha $(P I K 3 C A)$ mutation might account for nonresponders to EGFR targeting in CRC. ${ }^{6-8}$ For example, De Roock et $\mathrm{al}^{9}$ found that patients with $P I K 3 C A$ mutations had a significantly lower response rate in $K R A S$ wild-type patient.

Furthermore, in clinical practice, most results of gene tests were carried out mainly on primary tumors due to the difficulties to obtain tissues of metastatic tumors. However, some research results raise concerns that genetic profiling of primary tumors may not be representative of metastatic disease. ${ }^{10,11}$ In MRCRC, the concordance of gene mutation status between the primary tumors and metastases is controversial. Jones et $\mathrm{al}^{12}$ found a high degree of concordance between primary tumors and metastases. In contrast, Vermaat et $\mathrm{al}^{13}$ reported a high degree of mutational discordance between primary and metastatic samples using next-generation sequencing. Some study reports have shown gene mutations, such as $K R A S, N R A S$, and $B R A F$ were highly concordant between primary tumors and metastases, ${ }^{14,15}$ while discordant mutations were observed in genes of the phosphoinositide 3-kinase pathway.

Consideration of the above phenomenon, the heterogeneity between primary tumors and metastases seemed as an additional reason for the failure of targeted therapies in MRCRCs. Thus, our study was aimed to evaluate the genetic relationship between primary MRCRCs and their matched metastases that will consequently help in targeted therapy.

\section{Materials and methods Study population}

Among patients with histologically confirmed colorectal adenocarcinoma who had been treated or followed up at Zhejiang Cancer Hospital between June 2004 and July 2013, 59 MRCRC patients who had undergone surgical resection or biopsy of both primary tumors and related metastatic sites were enrolled. The retrospective study was performed using the stored samples at the Department of Pathology, and all of the samples were from patients who had received surgical resection or biopsy of both primary and related metastatic tumors with their consent, and anonymized before the study. The patient did not provide written informed consent in our study. The study was approved by the medical ethics committee at Zhejiang Cancer Hospital.
Table I The PCR primers for KRAS and PIK3CA gene amplification

\begin{tabular}{|c|c|}
\hline Gene & Primers \\
\hline \multicolumn{2}{|l|}{ KRAS } \\
\hline Codons & $\begin{array}{l}\text { 5'-AGGTACTGGTGGAGTATTTGATAGTGT-3' } \\
\text { (forward) }\end{array}$ \\
\hline 12,13 & 5'-CCTCTATTGTTGGATCATATTCGTC-3' (reverse) \\
\hline \multirow[t]{2}{*}{ Codons 61} & 5'-GGTGCACTGTAATAATCCAGACT-3' (forward) \\
\hline & 5'-CATGGCATTAGCAAAGACTCA-3' (reverse) \\
\hline Codons & 5'-AGACACAAAACAGGCTCAGGA-3' (forward) \\
\hline 117 & $\begin{array}{l}\text { 5'-TTGAGAGAAAAACTGATATATTAAATGAC-3' } \\
\text { (reverse) }\end{array}$ \\
\hline \multicolumn{2}{|l|}{ PIK3CA } \\
\hline Codons & 5'-AGACACAAAACAGGCTCAGGA-3' (forward) \\
\hline 545 & $\begin{array}{l}\text { 5'-TTGAGAGAAAAACTGATATATTAAATGAC-3' } \\
\text { (reverse) }\end{array}$ \\
\hline
\end{tabular}

Abbreviations: KRAS, Kirsten rat sarcoma viral oncogene homolog; PCR, polymerase chain reaction; PIK3CA, phosphatidylinositol-4,5-bisphosphate 3-kinase catalytic subunit alpha.

\section{Sequence analysis of KRAS and PIK3CA gene}

Formalin-fixed paraffin-embedded primary tumor and metastatic tissue specimens were microdissected manually under the supervision of experienced gastrointestinal pathologists. The DNA was extracted according to the manufacturer's instructions of E.Z.N.A.FFPE DNA Kit (Lot. D3399-01, OMEGA Bio-Tek, Norcross, GA, USA). For mutation analyses, extracted tumor DNA samples were amplified by polymerase chain reaction (PCR) using primers (Table 1). The PCR conditions were as follows: one cycle of $95^{\circ} \mathrm{C}$ for $5 \mathrm{~min}$ utes; 34 cycles of $95^{\circ} \mathrm{C}$ for 30 seconds, $58^{\circ} \mathrm{C}$ for 30 seconds, and $72^{\circ} \mathrm{C}$ for 1 minute; and one cycle of $72^{\circ} \mathrm{C}$ for 10 minutes. The PCR products were determined by $3 \%$ agarose gel electrophoresis and then sequenced using the same forward primer of each gene by Invitrogen 3730XL genetic analyzer (Life Technologies, Carlsbad, CA, USA). The sequencing results were analyzed with Chromas software under the condition of signal-to-noise ratio $>98 \%$.

\section{Statistical analysis}

The concordant rate of $K R A S$ and $P I K 3 C A$ mutational status in primary tumors and related metastases was evaluated, the Kappa index was measured using Cohen's kappa coefficient, which can assess the concordance between categorical variables of the same individuals. ${ }^{16}$ The effect of $K R A S$ and PIK3CA mutational status on clinicopathologic features was assessed using Pearson's chi-square or Fisher's exact tests. The relationship between the discordant rates of the KRAS and $P I K 3 C A$ mutation status and various clinicopathologic 
features was also evaluated using univariate analyses (Pearson's chi-square or Fisher's exact tests). $P<0.05$ was considered significant. All analyses were performed using SPSS for Windows, version 18.0 (SPSS Inc., Chicago, IL, USA).

\section{Results}

\section{Patient characteristics $(\mathrm{N}=59)$ and the association between KRAS or PIK3CA mutational status and clinicopathologic features}

Of the 59 MRCRC in this study, 30 patients (50.8\%) were male. The median age was 46 years (range, $18-80$ years). Related metastatic sites for gene analysis were liver $(\mathrm{N}=33)$, lung $(\mathrm{N}=9)$, peritoneum $(\mathrm{N}=4)$, pelvic $(\mathrm{N}=4)$, ovary/uterus $(\mathrm{N}=6)$, groin $(\mathrm{N}=1)$, stomas $(\mathrm{N}=1)$, and pancreas $(\mathrm{N}=1)$, respectively. The primary tumor sites were right colon in 13 patients $(22.0 \%)$, left colon in 15 patients $(25.4 \%)$, rectum in 29 patients $(49.2 \%)$, and others in two patients (3.4\%). Other patient characteristics are shown in Table 2. Age in MRCRC patients was different according to the KRAS mutational status. Age $<60$ was more frequent in wild-type $K R A S$ patients than in mutant KRAS patients $(83.3 \%$ vs
$16.7 \% ; P=0.010)$. There was no association between the KRAS or PIK3CA status and other clinicopathologic features (sex, primary tumor location, histology and grade, clinical situations for the development of systemic metastasis, and metastasis sites) (Table 2).

\section{Frequency and types of KRAS and PIK3CA mutation}

Ten (16.9\%) patients with KRAS mutation and 26 (44.1\%) patients with PIK3CA mutation were observed in primary tumors, with eleven (18.6\%) and $32(54.2 \%)$ in related metastatic sites, respectively. Seventeen patients $(28.8 \%)$ had the KRAS mutation and 46 patients (80.0\%) had PIK3CA mutation in any place of the primary or metastatic sites. Of those 17 patients with KRAS mutation, two patients had a KRAS12 codon GGT-AGT mutation, five had a KRAS12 codon GGT-GCT mutation, four had a KRAS12 codon GGT-GAT mutation, three had a KRAS12 codon GGT-GTT mutation, two had a KRAS13 codon GGC-GAC mutation (one combined with KRAS12 mutation), one had a KRAS61 codon CAA-CAT mutation, and one patient had a KRAS117 codon AAA-ATA mutation. In addition, of those 46 patients with PIK3CA mutation, 45 patients had a PIK3CA545 codon

Table 2 Patient characteristics and the association between KRAS or PIK3CA status and clinicopathologic parameters

\begin{tabular}{|c|c|c|c|c|c|c|c|}
\hline \multirow[t]{4}{*}{ Characteristic } & \multicolumn{7}{|c|}{ Number of patients } \\
\hline & \multirow{3}{*}{$\begin{array}{l}\text { All } \\
\mathbf{N}\end{array}$} & \multirow{2}{*}{$\frac{\text { KRAS }}{\text { WT }}$} & \multirow{2}{*}{$\frac{K R A S}{M T}$} & \multirow[t]{3}{*}{$P$-value } & \multirow{2}{*}{$\frac{\text { PIK3CA }}{\text { WT }}$} & \multirow{2}{*}{$\frac{\text { PIKЗCA }}{M T}$} & \multirow[t]{3}{*}{$P$-value } \\
\hline & & & & & & & \\
\hline & & $\mathbf{N}(\%)$ & $\mathbf{N}(\%)$ & & $\mathbf{N}(\%)$ & $\mathbf{N}(\%)$ & \\
\hline Sex & & & & 0.054 & & & 0.701 \\
\hline Male & 30 & $18(60.0)$ & $12(40.0)$ & & $6(20.0)$ & $24(80.0)$ & \\
\hline Female & 29 & $24(82.8)$ & $5(17.2)$ & & $7(24.1)$ & $22(75.9)$ & \\
\hline Age & & & & 0.010 & & & 0.548 \\
\hline$<60$ years & 36 & $30(83.3)$ & $6(16.7)$ & & $7(19.4)$ & $29(80.6)$ & \\
\hline$\geq 60$ years & 23 & $12(52.2)$ & II (47.8) & & $6(26.1)$ & $17(73.9)$ & \\
\hline Primary tumor location & & & & 0.777 & & & 0.563 \\
\hline Right & 13 & $9(69.2)$ & $4(30.8)$ & & $2(15.4)$ & II (84.6) & \\
\hline Left & 15 & $12(80.0)$ & $3(20.0)$ & & $5(33.3)$ & $10(66.7)$ & \\
\hline Rectum & 29 & $20(70.0)$ & $9(30.0)$ & & $6(20.7)$ & $23(79.3)$ & \\
\hline Others & 2 & I (50.0) & I (50.0) & & $0(0)$ & $2(100)$ & \\
\hline Metastasis & & & & 0.088 & & & 0.410 \\
\hline Synchronous & 24 & $20(83.3)$ & $4(16.7)$ & & $4(16.7)$ & $20(83.3)$ & \\
\hline Metachronous & 35 & $22(62.9)$ & $13(37.1)$ & & $9(25.7)$ & $26(74.3)$ & \\
\hline Histology and grade & & & & 0.885 & & & 0.787 \\
\hline Well/moderately differentiated & 39 & $28(71.8)$ & II (28.2) & & $9(23.1)$ & $30(76.9)$ & \\
\hline Poorly differentiated, mucinous & 20 & $14(70.0)$ & $6(30.0)$ & & $4(20.0)$ & $16(80.0)$ & \\
\hline Resection style & & & & 0.770 & & & 0.584 \\
\hline Concurrent & 19 & 14 (73.7) & $5(26.3)$ & & $5(26.3)$ & 14 (73.7) & \\
\hline Subsequent & 40 & $28(70.0)$ & $12(30.0)$ & & $8(20.0)$ & $32(80.0)$ & \\
\hline
\end{tabular}

Abbreviations: KRAS, Kirsten rat sarcoma viral oncogene homolog; PIK3CA, phosphatidylinositol-4,5-bisphosphate 3-kinase catalytic subunit alpha; WT, wild-type; MT, mutation type. 
Table 3 Distribution of KRAS mutation types

\begin{tabular}{llll}
\hline Gene & Mutation types & Patients $(\mathbf{N})$ & $\%$ \\
\hline KRAS (28.8\%) & KRAS I2 codon GGT-ACT & 2 & 11.76 \\
& KRAS I2 codon GGT-GCT & 5 & 29.4 I \\
& KRAS I2 codon GGT-GAT & 4 & 25.53 \\
& KRAS I2 codon GGT-GTT & 3 & 17.65 \\
& KRAS I3 codon GGC-GAC & $2 *$ & 5.88 \\
& KRAS 6 I codon CAA-CAT & I & 5.88 \\
& KRAS I I7 codon AAA-ATA & I & 5.88 \\
PIK3CA (80.0\%) & PIK3CA 545 codon CAG-GCG & 45 & 97.83 \\
& PIK3CA 545 codon CAG-AAG & I & 2.17 \\
\hline
\end{tabular}

Note: *One patient combined with KRAS 12 mutation.

Abbreviations: KRAS, Kirsten rat sarcoma viral oncogene homolog; PIK3CA, phosphatidylinositol-4,5-bisphosphate 3-kinase catalytic subunit alpha.

CAG-GCG mutation and one had a PIK3CA 545 codon CAG-AAG mutation (Table 3).

\section{Concordance of KRAS and PIK3CA status in primary tumors and related metastases}

$K R A S$ status was concordant between primary and metastatic sites in 45 patients $(76.3 \%$; kappa $=0.157)$. Of the 14 discordant cases, seven patients had the KRAS mutation in the primary tumors, but not in the metastatic sites; seven patients had the $K R A S$ mutation only in the metastatic tumors and not in the primary tumors. PIK3CA status was low concordant between primary and metastatic sites in 25 patients (42.4\%; kappa $=-0.141)$. Of the 34 discordant cases, 14 patients had the $K R A S$ mutation in the primary tumors, and not in the metastatic sites; 20 patients had the $K R A S$ mutation in the metastatic tumors without in the primary tumors (Table 4 ).

\section{Discordance rates of KRAS and PIK3CA} status according to various clinicopathologic features

We evaluated the discordance rates of $K R A S$ and $P I K 3 C A$ mutation status between primary tumors and paired tissues

Table 4 KRAS and PIK3CA mutational status of primary tumors and paired metastatic sites

\begin{tabular}{lll}
\hline Primary sites & Metastatic sites \\
\cline { 2 - 3 } & No of wild-type & No of mutation type \\
\hline KRAS & & 7 \\
No of wild-type & 42 & 3 \\
No of mutation type & 7 & \\
PIK3CA & & 20 \\
No of wild-type & 13 & 12 \\
No of mutation type & 14 & \\
\hline
\end{tabular}

Abbreviations: KRAS, Kirsten rat sarcoma viral oncogene homolog; PIK3CA, phosphatidylinositol-4,5-bisphosphate 3-kinase catalytic subunit alpha; No, number. with various clinicopathologic factors such as primary tumor location, metastatic organs, type of primary or metastatic tumor specimens (biopsied vs resected), and any chemotherapy/ radiotherapy between resection of primary and metastasis before obtaining primary or metastatic tumor specimens or resection style. The lung was the most frequent site showing $K R A S$ discordance, however, no difference was seen in the discordance rate of KRAS status for the metastatic organs. Two patients with tissues acquired from primary tumor (left colon and rectal colon) and related stomas and groin metastatic site showed discordance of $K R A S$ status (mutation type $[\mathrm{P}]$-wild-type $[\mathrm{M}])$. One patient with tissues acquired from primary tumor (transverse colon) and related pancreatic metastatic site showed concordance of PIK3CA status (mutation type $[\mathrm{P}]$-mutation type $[\mathrm{M}])$. Otherwise, the discordant rate of PIK3CA status was frequent (liver, 60.6\%; lung, 55.5\%; and peritoneum, $50.0 \%$ ) regardless of related metastatic site. Two patients with tissues acquired from primary tumor (transverse colon and left colon) and related pancreatic and stomas metastatic site showed discordance of PIK3CA status (mutation type $[\mathrm{P}]$-wild-type $[\mathrm{M}]$ ). One patient with tissues acquired from primary tumor (rectal colon) and related groin metastatic site showed concordance of PIK3CA status (mutation type $[\mathrm{P}]$-wild-type $[\mathrm{P}])$. Additional analyses were performed to find whether the discordance rates of KRAS and PIK3CA status were influenced by other various clinicopathologic factors $(67.5 \%$ vs $36.8 \% ; P=0.026)$. However, there was no difference in the discordant rate of $K R A S$ and $P I K 3 C A$ status for other clinicopathologic factors (Table 5).

\section{Discussion}

EGFR monoclonal antibodies, such as cetuximab and panitumumab are currently approved for the treatment of metastatic CRC patients with KRAS wild-type tumors. However, their antitumor activity has been limited by intrinsic and acquired drug resistance. One explanation for drug resistance is cancer genetic heterogeneity, which contains content of two aspects. ${ }^{13,17}$ One refers CRC patients can harbor different gene mutations between primary tumors and paired metastatic sites, another means that even gene disaccordance existed within different regions of the same tissues. Heterogeneous in genes have been reported to play a role in resistance to anti-EGFR drugs in CRC, including activating mutations in $K R A S, N R A S, B R A F$, and $P I K 3 C A .{ }^{9}$ Genetic heterogeneity makes it more difficult to decide to use the anti-EGFR drugs only based on the results of gene test either from primary or metastatic tumor. Therefore, we conducted the study to compare these gene statuses between 
Table 5 Univariate analyses on the association between clinicopathologic features and the discordance rates of KRAS and PIK3CA mutation status

\begin{tabular}{|c|c|c|c|c|}
\hline \multirow[t]{2}{*}{$\overline{\text { Gene }}$} & \multirow[t]{2}{*}{ Characteristic } & \multicolumn{3}{|l|}{ Univariate analysis } \\
\hline & & $\begin{array}{l}\text { Concordant cases, } \\
\text { N (\%) }\end{array}$ & $\begin{array}{l}\text { Discordant cases, } \\
\text { N (\%) }\end{array}$ & $P$-value \\
\hline & Metastatic site & & & $0.27 I$ \\
\hline & Liver & $25(75.8)$ & $8(24.2)$ & \\
\hline & Lung & $6(66.7)$ & $3(33.3)$ & \\
\hline & Peritoneum/ovary/uterus/pelvic & $13(92.9)$ & I (7.I) & \\
\hline & Others & I (33.3) & $2(66.7)$ & \\
\hline & Primary tumor location & & & 0.677 \\
\hline & Right & $9(90)$ & $I(10)$ & \\
\hline & Left & II (78.6) & $3(21.4)$ & \\
\hline & Rectum & $21(72.4)$ & $8(27.6)$ & \\
\hline \multirow[t]{19}{*}{ KRAS } & Type of tumor specimens & & & 0.095 \\
\hline & Resected & $32(7 I .1)$ & $13(28.9)$ & \\
\hline & Biopsied & $13(92.8)$ & $\mathrm{I}(7.1)$ & \\
\hline & Any chemotherapy/radiotherapy between resection & & & 0.333 \\
\hline & of primary and metastasis & & & \\
\hline & Yes & $29(80.6)$ & $7(19.4)$ & \\
\hline & No & $16(69.6)$ & $7(30.4)$ & \\
\hline & Resection style & & & 0.748 \\
\hline & Synchronous & $14(73.7)$ & $5(26.3)$ & \\
\hline & Metachronous & $31(77.5)$ & $9(22.5)$ & \\
\hline & Metastatic site & & & 0.794 \\
\hline & Liver & I3 (39.4) & $20(60.6)$ & \\
\hline & Lung & $4(44.4)$ & $5(55.6)$ & \\
\hline & Peritoneum/ovary/uterus/pelvic & $7(50.0)$ & $7(50.0)$ & \\
\hline & Others & I (47.I) & $2(52.9)$ & \\
\hline & Primary tumor location & & & 0.606 \\
\hline & Right & $3(30)$ & $7(70)$ & \\
\hline & Left & $7(50)$ & $7(50)$ & \\
\hline & Rectum & $13(44.8)$ & $16(55.2)$ & \\
\hline \multirow[t]{10}{*}{ PIKЗCA } & Type of tumor specimens & & & 0.564 \\
\hline & Resected & $20(45.4)$ & $25(55.6)$ & \\
\hline & Biopsied & $5(35.7)$ & $9(64.3)$ & \\
\hline & Any chemotherapy/radiotherapy between resection & & & 0.223 \\
\hline & of primary and metastasis & & & \\
\hline & Yes & $13(36.1)$ & $23(63.9)$ & \\
\hline & No & $12(52.2)$ & II (47.8) & \\
\hline & Resection style & & & 0.026 \\
\hline & Synchronous & $12(63.2)$ & $7(36.8)$ & \\
\hline & Metachronous & $13(32.5)$ & $27(67.5)$ & \\
\hline
\end{tabular}

Abbreviations: KRAS, Kirsten rat sarcoma viral oncogene homolog; PIK3CA, phosphatidylinositol-4,5-bisphosphate 3-kinase catalytic subunit alpha.

primary tumors and paired metastasis in CRC for developing effective therapeutic strategies. We evaluated all the genes statuses, such as KRAS, NRAS, BRAF, and PIK3CA in primary $C R C$ and their matched metastasis, and compared the discordance between the two sites. However, only one patient with NRAS mutation was found in metastatic site and no $B R A F$ mutation was detected due to the relatively small sample size. Subsequently, we performed direct sequencing of KRAS and PIK3CA genes in 59 primary CRC tumors and matched metastases to define the mutational concordance of these genes in primary and metastatic tumors. The KRAS or PIK3CA mutational statuses were regarded as positive if they were mutated in any place of primary tumors or related metastases in our study. Approximately $28.8 \%$ of CRCs harbor KRAS mutations, which is almost consistent with prior studies. While PIK3CA mutations were detected in $80 \%$ of patients with $44.1 \%$ of primary tumors and $54.2 \%$ of related metastatic sites, respectively, which showed relatively higher mutational rate than that of prior reports. ${ }^{18,19}$ Approximately more than $80 \%$ KRAS mutations were detected in codons 12,13 in exon 2, and our results showed $100 \%$ mutation analyses in codons 545 of the PIK3CA gene 
in exon 9. Furthermore, ten patients out of 59 in our study harbored both KRAS and PIK3CA mutations, which may be a certain cluster having the low response to EGFR-targeted treatment and a poor prognosis, which can be deduced from the previous reports.

Our findings demonstrate a high level of concordance of $K R A S$ mutation status and a significant discordance of $P I K 3 C A$ mutation status in primary tumors and matched metastases, which is in agreement with previous studies. ${ }^{20,21}$ $K R A S$ status was found to be concordant in $76.3 \%$ of the analyzed primary and metastatic sites in 59 patients, while PIK3CA gene discordant rate was up to $57.6 \%$ in our study. As the sample size was small $(\mathrm{N}=59)$, we did not find difference of gene mutational rate of $K R A S$ and $P I K 3 C A$ in the primary tumors and metastasis in these 59 patients. Nevertheless, $P I K 3 C A$ gene mutational rate was more frequently observed in metastatic specimens than primary sites $(61.5 \%$ vs $44.6 \% ; P=0.025$ ) in our unselected CRC patients (table not shown). De Roock et $\mathrm{al}^{9}$ reported that $P I K 3 C A$ mutations may negatively impact the response to EGFR inhibitors of CRC patients, and PIK3CA exon 20 mutations were also significantly associated with shorter survival. ${ }^{7}$ Also, Domingo et $\mathrm{al}^{22}$ found that patients with $P I K 3 C A$ mutant could benefit from aspirin therapy after CRC diagnosis and had a reduced rate of CRC recurrence. Therefore, our findings indicated that if patients with wild-type $K R A S$ were selected to receive EGFRtargeted therapy, it could be more appropriate to perform $K R A S$ and PIK3CA genotyping in both the primary tumors and metastases. Baldus et $\mathrm{al}^{21}$ previously reported the obvious discordance of the status of gene involved in PIK3CA/ AKT pathway between primary tumors and metastasis, also with relative higher mutational rate in metastatic sites. Moreover, acquisition of new mutations may be developed during the evolution of the metastatic process. ${ }^{23}$ It is obvious that it remains to be proven in prospective well-designed clinical studies.

We also discussed the main influencing factors resulting in genetic heterogeneity between primary and metastatic tissues. As previously reported, discrepancy may be related to the different sites of primary tumor location, the metastatic organs, type of tumor specimens (resected or biopsied), any therapy between resection of primary and metastasis or resection style (synchronous or metachronous) (Goswami et al, ${ }^{23}$ 2014 ASCO Annual Meeting). However, in our study, we found no difference in the discordant rate of $K R A S$ and $P I K 3 C A$ status with these clinicopathologic factors except the paired specimens obtained from metachronous resection showed an increased $P I K 3 C A$ discordant rate compared with synchronous resection. Goswami et al reported that metachronous resection of the two sites, receiving intervening chemotherapy between resection of primary and metastasis and even increasing number of lines of intervening chemotherapy, can be the possibility for the observed discordance in the KRAS and PIK3CA mutation status. ${ }^{23}$ The rates of $K R A S$ and $P I K 3 C A$ gene discordance between primary and metastasis vary by the related metastatic sites, with a relatively higher rate of discordance in brain, bone, peritoneum, or lung metastases when compared with other metastatic organs including liver, distant $\mathrm{LN}$, or ovary. ${ }^{24}$ Therefore, the causes of discordance need to be further evaluated in future larger studies.

Our study shares several limitations common to the majority of published findings in this field. First, our mutational analysis was performed by the traditional sequencing analysis with relatively low sensitivity, and not confirmed by other more sensitive methods as amplification-refractory mutation system allele-specific PCR combined with Scorpions probes or peptide nucleic acid-clamp allelespecific reverse transcription-PCR assay. Second, the retrospective analysis is prone to bias or error, and the sample is relatively small. Third, we did not conduct the repetitive and multipoint mutational analysis of the same tissue, which may lead to the false-negative results. Despite these limitations, our study provides some clinically meaningful suggestions. This study demonstrated the existence of a significant discordance of PIK3CA and relative concordance of KRAS mutations occurring in primary tumors and their corresponding metastases in patients with CRC. These reminded that gene test can not only be conducted in primary tissues, but the metastatic specimen also needs to be reexamined if the tissue is available. Our study also raised the hypothesis that combined analysis of $K R A S$ and PIK3CA to select the proper CRC patients will be an effective strategy for EGFR-targeted therapy, which remains to be proven in well-designed clinical studies.

In conclusion, our findings indicate a concordance of $K R A S$ mutation and a discordance of PIK3CA mutation between the primary tumors and the matched metastases in CRC and suggest that status of specific molecules in metastatic tumors need to be reevaluated when the patients with metastases are about to use the EGFR-targeted therapy.

\section{Acknowledgment}

This work was supported by Natural Science Foundation of Zhejiang Province (No LY14H160008). 


\section{Author contributions}

All authors contributed toward data analysis, drafting and revising the paper and agree to be accountable for all aspects of the work.

\section{Disclosure}

The authors report no conflicts of interest in this work.

\section{References}

1. Jemal A, Siegel R, Xu J, Ward E. Cancer statistics, 2010. CA Cancer J Clin. 2010;60:277-300.

2. Morton RF, Hammond EH. ASCO provisional clinical opinion: KRAS, cetuximab, and panitumumab-clinical implications in colorectal cancer. J Oncol Pract. 2009;5:71-72.

3. Perkins G, Lievre A, Ramacci C, et al. Additional value of EGFR downstream signaling phosphoprotein expression to KRAS status for response to anti-EGFR antibodies in colorectal cancer. Int J Cancer. 2010; 127:1321-1331.

4. Karapetis CS, Khambata-Ford S, Jonker DJ, et al. K-ras mutations and benefit from cetuximab in advanced colorectal cancer. $N$ Engl J Med. 2008;359:1757-1765.

5. Lievre A, Bachet JB, Boige V, et al. KRAS mutations as an independent prognostic factor in patients with advanced colorectal cancer treated with cetuximab. J Clin Oncol. 2008;26:374-379.

6. Wong NS, Fernando NH, Nixon AB, et al. A phase II study of capecitabine, oxaliplatin, bevacizumab and cetuximab in the treatment of metastatic colorectal cancer. Anticancer Res. 2011;31:255-261.

7. Perrone F, Lampis A, Orsenigo M, et al. PI3KCA/PTEN deregulation contributes to impaired responses to cetuximab in metastatic colorectal cancer patients. Ann Oncol. 2009;20:84-90.

8. Sartore-Bianchi A, Martini M, Molinari F, et al. PIK3CA mutations in colorectal cancer are associated with clinical resistance to EGFRtargeted monoclonal antibodies. Cancer Res. 2009;69:1851-1857.

9. De Roock W, Claes B, Bernasconi D, et al. Effects of KRAS, BRAF, NRAS, and PIK3CA mutations on the efficacy of cetuximab plus chemotherapy in chemotherapy-refractory metastatic colorectal cancer: a retrospective consortium analysis. Lancet Oncol. 2010;11:753-762.

10. Bedard PL, Hansen AR, Ratain MJ, Siu LL. Tumour heterogeneity in the clinic. Nature. 2013;501:355-364.

11. Gerlinger M, Rowan AJ, Horswell S, et al. Intratumor heterogeneity and branched evolution revealed by multiregion sequencing. $N$ Engl J Med. 2012;366:883-892.
12. Jones S, Chen WD, Parmigiani G, et al. Comparative lesion sequencing provides insights into tumor evolution. Proc Natl Acad Sci U S A. 2008; $105: 4283-4288$.

13. Vermaat JS, Nijman IJ, Koudijs MJ, et al. Primary colorectal cancers and their subsequent hepatic metastases are genetically different: implications for selection of patients for targeted treatment. Clin Cancer Res. 2012;18:688-699.

14. Brannon AR, Vakiani E, Sylvester BE, et al. Comparative sequencing analysis reveals high genomic concordance between matched primary and metastatic colorectal cancer lesions. Genome Biol. 2014;15:454.

15. Vakiani E, Janakiraman M, Shen R, et al. Comparative genomic analysis of primary versus metastatic colorectal carcinomas. J Clin Oncol. 2012; 30:2956-2962.

16. Landis JR, Koch GG. The measurement of observer agreement for categorical data. Biometrics. 1977;33:159-174.

17. Lee SY, Haq F, Kim D, et al. Comparative genomic analysis of primary and synchronous metastatic colorectal cancers. PLoS One. 2014 9:e90459.

18. Foltran L, De Maglio G, Pella N, et al. Prognostic role of KRAS, NRAS, BRAF and PIK3CA mutations in advanced colorectal cancer Future Oncol. 2015;11:629-640.

19. Imamura $Y$, Lochhead $P$, Yamauchi M, et al. Analyses of clinicopathological, molecular, and prognostic associations of KRAS codon 61 and codon 146 mutations in colorectal cancer: cohort study and literature review. Mol Cancer. 2014;13:135.

20. Baas JM, Krens LL, Guchelaar HJ, Morreau H, Gelderblom H. Concordance of predictive markers for EGFR inhibitors in primary tumors and metastases in colorectal cancer: a review. Oncologist. 2011;16: 1239-1249.

21. Baldus SE, Schaefer KL, Engers R, Hartleb D, Stoecklein NH, Gabbert HE. Prevalence and heterogeneity of KRAS, BRAF, and PIK3CA mutations in primary colorectal adenocarcinomas and their corresponding metastases. Clin Cancer Res. 2010;16:790-799.

22. Domingo E, Church DN, Sieber O, et al. Evaluation of PIK3CA mutation as a predictor of benefit from nonsteroidal anti-inflammatory drug therapy in colorectal cancer. J Clin Oncol. 2013;31:4297-4305.

23. Goswami RS, Patel KP, Singh RR, et al. Hotspot mutation panel testing reveals clonal evolution in a study of 265 paired primary and metastatic tumors. Clin Cancer Res. 2015;21:2644-2651.

24. Kim MJ, Lee HS, Kim JH, et al. Different metastatic pattern according to the KRAS mutational status and site-specific discordance of KRAS status in patients with colorectal cancer. BMC Cancer. 2012;12:347.
OncoTargets and Therapy

\section{Publish your work in this journal}

OncoTargets and Therapy is an international, peer-reviewed, open access journal focusing on the pathological basis of all cancers, potential targets for therapy and treatment protocols employed to improve the management of cancer patients. The journal also focuses on the impact of management programs and new therapeutic agents and protocols on

\section{Dovepress}

patient perspectives such as quality of life, adherence and satisfaction. The manuscript management system is completely online and includes a very quick and fair peer-review system, which is all easy to use. Visit http://www.dovepress.com/testimonials.php to read real quotes from published authors. 